\title{
Novelty Determines the Effects of Olfactory Enrichment on Memory and Neurogenesis Through Noradrenergic Mechanisms
}

\author{
Alexandra Veyrac ${ }^{1,2}$, Joëlle Sacquet ${ }^{1,2}$, Véronique Nguyen ${ }^{3}$, Marc Marien $^{3}$, François Jourdan ${ }^{1,2}$ and \\ Anne Didier*, 1,2 \\ 'Université de Lyon, Lyon F-69007, Université Lyon I, F-69007, Lyon, CNRS-UMR 5020, Neurosciences Sensorielles, Comportement, Cognition, \\ CNRS-UMR 5020, Université Claude Bernard, Lyon, France; ${ }^{2}$ Institut Fédératif des Neurosciences de Lyon, Lyon, France; ${ }^{3}$ Division Neurobiologie I, \\ Centre de Recherche Pierre Fabre, Castres, France
}

\begin{abstract}
Commonly used experimental paradigms of environmental enrichment combine increased social interactions and sensory inputs and renewal of the objects present in the environment. However, the specific contribution of novelty to the effects of enrichment is unclear. Here, we show that repeated daily exposure to single novel odorants and not to an enriched but stable olfactory environment improves short-term olfactory memory and neurogenesis in the mouse olfactory bulb. In addition, these positive effects are mediated by noradrenalin as they are blocked by a noradrenergic receptor antagonist. These data suggest that novelty recognition and noradrenergic mechanisms are crucial in mediating neural plasticity induced by olfactory enrichment.
\end{abstract}

Neuropsychopharmacology (2009) 34, 786-795; doi: I0.1038/npp.2008.191; published online 22 October 2008

Keywords: enrichment; olfaction; noradrenalin; neurogenesis; learning; mouse

\section{INTRODUCTION}

Environmental enrichment is a commonly used experimental paradigm known to improve brain function through the enhancement of neuronal plasticity (Bennett et al, 1969; Rosenzweig and Bennett, 1996), learning, and neurogenesis in the adult brain (van Praag et al, 2000). Environmental enrichment in rodents is provided by housing conditions that favor social interactions with congeners, exploration of various toys, and permits repeated exposures to novelty through the renewal of the items that make up the enriched environment. The understanding of the mechanisms underlying the beneficial effects of enrichment is hampered by the difficulty in identifying those components of enrichment that actually contribute to these effects. Although attempts have been made to isolate the role of some factors such as social interactions (Schrijver et al, 2002, 2004) or physical exercise (van Praag et al, 1999), the contribution of novelty to the positive effects of enrichment on neuronal plasticity and brain function has not been specifically investigated (van Praag et al, 2000; Nithianantharajah and Hannan, 2006). Our hypothesis is that exposure to novelty may have

*Correspondence: Dr A Didier, Université Lyon I, UMR CNRS 5020, 50 Avenue Tony Garnier, F-69366 Lyon, France. Tel: + 33043728 74 99, Fax: + 3304372876 01, E-mail: didier@olfac.univ-lyon I.fr Received 2 June 2008; revised 19 September 2008; accepted 19 September 2008 functional and cellular outcomes distinct from those induced by an enriched but stable environment, because the perception of novelty relies on specific networks involving noradrenergic neurotransmission (Foote et al, 1980; Aston-Jones and Bloom, 1981; Vankov et al, 1995). To explore this hypothesis, we took advantage of the wellknown functional organization of the olfactory bulb (OB), a forebrain region that (1) is a highly plastic structure that participates in elaborating olfactory memory, (2) harbors a well-established and intense level of neurogenesis in the adult brain (Lledo et al, 2006), (3) receives sensory inputs directly from olfactory receptor neurons located in the nasal cavity, thereby allowing experimental control of the nature and level of incoming olfactory information, and (4) receives strong noradrenergic innervations from the locus coeruleus (Shipley et al, 1985).

\section{MATERIALS AND METHODS}

\section{Animals}

Adult male C57/BL6J mice (Charles River Laboratories, Domaine des Oncins, France) aged 8-12 weeks at the beginning of the experiment were used $(n=96)$. The animals were housed under a 12-h light/dark cycle with food and water available ad libitum. All efforts were made to minimize the number of animals used and their suffering during experimental procedure in accordance with the 
European Community Council Directive 86/609/EEC, and in compliance with French regulations and local ethical committee guidelines for animal research.

\section{Housing Conditions and Enrichment Protocols}

Mice were housed in groups of six in standard laboratory cages $(43 \times 15 \times 18 \mathrm{~cm})$ covered with acrylic filtering lids. Mice were randomly assigned to four experimental groups ( $n=23-29$ mice per group). In the olfactory enrichment/ novelty group (OEN), mice were exposed to a single odor placed in a tea ball suspended from the cage lid and replaced every $24 \mathrm{~h}$. The olfactory enrichment/stable group (OES) was housed in similar conditions except that all the odors were blended in the tea ball. The mixture was composed of a small sample of each odorant and was prepared fresh every 2 days. A third experimental group (OEN-Lab) was similar to the OEN group but received in addition a treatment with labetalol, a mixed $\beta$ - and $\alpha 1$ adrenoceptor antagonist, delivered in the drinking water as detailed below. Control mice were housed under the same conditions (same cage and same number of animals per cage) but with empty tea balls. All enrichment paradigms and drug treatment (labetalol) lasted 47 days (Figure 1a). Enriched and standard animals were kept in separate rooms.

\section{Odor Set}

Twenty different odors were used for olfactory enrichment: pepper, star anise, fennel, cinnamon, garlic, onion, ginger, juniper berries, clove, nutmeg, lemon, celery, cumin, chocolate, cardamom, thyme, tarragon, capsicum, lavender, and orange. In the groups with daily renewal of the odorants (OEN and OEN-Lab), the same sequence of odorant presentation was repeated after 20 days. In the OES group, the 20 odors were blended.

\section{Labetalol Delivery}

On the basis of the measure of the daily water intake of mice in our animal facility $(5.43 \pm 0.24 \mathrm{ml}$ per day per animal), the concentration of labetalol (Sigma, Saint Louis, MO, USA) in the drinking water was prepared so that the daily intake of drug would be $25 \mathrm{mg} / \mathrm{kg}$. The solution of labetalol was made fresh every day. The volume of the drug solution consumed was monitored daily and the effective drug dose intake by the animals was calculated a posteriori to be $22.4 \pm 2.9 \mathrm{mg} / \mathrm{kg}$ per day.

\section{Behavioral Experiments}

Experimental setup. All behavioral experiments were conducted on a specially designed computer-assisted twohole board apparatus for mice $(40 \times 40 \mathrm{~cm})$ (Mandairon et al, 2006d). The holes were odorized with $(+)$ limonene or $(-)$ carvone diluted $1: 10$ in mineral oil, placed on a polypropylene swab at the bottom of the hole and covered with cage bedding (wood shavings).

Odor recognition task. On day 42 of the experiment, mice were habituated to the experimental setup by a 2-min trial on the hole board, with no odor. After 2 days, mice were submitted to the odor recognition task, which consisted of two trials of 2-min duration each (Figure 1b). During the first (acquisition) trial, an unfamiliar odor ' $A$ ' $((+)$ limonene or $(-)$ carvone) was presented in the two holes of the board. After $60 \mathrm{~min}$, mice were subjected to the second (recall) trial in which one hole was odorized with the previously presented odor ' $A$ ' and the other hole with the new odor ' $B$ ' $((+)$ limonene or $(-)$ carvone). The odorants ' $A$ ' and ' $B$ ' were randomized between the acquisition and recall trials. To avoid a place preference bias, odors were randomly placed in one or the other hole. We previously verified in a separate group of mice that the two odorants were equally explored when presented together in a onetrial test, indicating that there was no preference of the mice for either odor (data not shown). The number of nose pokes into the holes (visits) and the time spent exploring the holes were automatically detected with capacitive sensors and recorded through custom-made computer software.

Data analysis and statistics. Mice with exploration time or number of visits exceeding group mean $\pm 2 S D$ were excluded from the analysis (two in Stand, three in OEN, two in OES, and two in OEN-Lab).

Acquisition trial: investigation time and number of visits (entries) in the two holes were averaged within each group. Between-group comparisons were carried out by ANOVA followed by Bonferroni post hoc tests (Systat 7.0.1 software; SSI, Richmond, CA, USA).

Recall trial: for each experimental group, investigation times of the novel or familiarized odors were compared by unilateral paired $t$-tests. The same was carried out for analysis of the number of visits to the novel or familiar odor. F-tests for assessment of equality of variance and Kruskal-Wallis ANOVA were also applied to these data, to assess between-group differences in overall exploration of the holes. In addition, an index of preference for novel odor was calculated as $\mathrm{Tn} /(\mathrm{Tn}+\mathrm{Tf}) \times 100$ where $\mathrm{Tn}$ is the time spend exploring the novel odor and $\mathrm{Tf}$ is the time spend exploring the familiarized odor. If mice remembered the first presented odor, they spent a greater percentage of time during recall exploring the hole containing the novel odor. If they did not remember the first presented odor, both holes were equally explored during recall. Significant exploration of the novel odor above $50 \%$ was assessed by one-sample $t$-tests (Veyrac et al, 2007).

Measurements of locomotor activity. During the habituation trial (2 days before the odor recognition task, see above) general locomotor activity of mice was measured with a video-tracking system (Viewpoint, Champagne au Mont d'Or, France). Between-group comparisons were carried out by ANOVA followed by Bonferroni post hoc tests.

\section{Measurement of Monoamine Neurotransmitters}

On day 47, 24 mice ( 6 per group) were killed by high-power head-focused microwave irradiation $(5.0 \mathrm{~kW}, 2450 \mathrm{MHz}$, $0.9 \mathrm{~s} ; \operatorname{Sacron}^{\mathrm{TM}}$ Model 8000; SAIREM, Vaulx-en-Velin, France). This microwaving procedure provides a rapid, noninvasive, and complete fixation (heat inactivation of enzymes) of the brain in situ, so that accurate determina- 


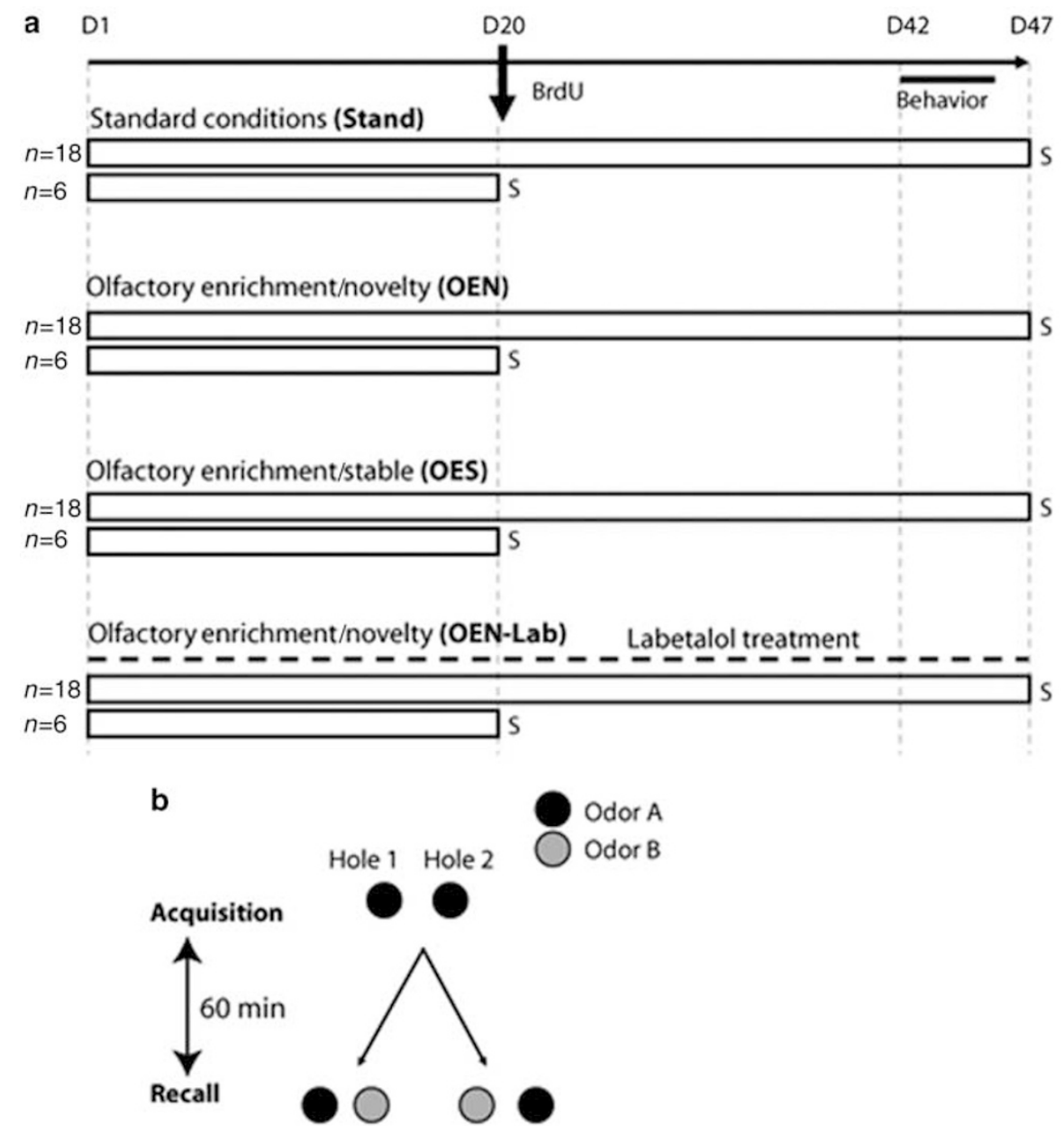

Figure I Experimental design. (a) Animals were housed either in standard conditions or in three different enrichment conditions for 47 days as described. Animals were injected with 5-bromo-2'deoxyuridine (BrdU) on day 20, and were killed $2 \mathrm{~h}$ afterwards for assessing proliferation in the subventricular zone, or 27 days later for assessing survival of newborn cells in the olfactory bulb (OB). Behavioral testing was carried out during the last week of the experiment. (b) The two-trial olfactory recognition task was conducted on a two-hole board apparatus adapted for mice, with a 60-min delay between acquisition and recall. During acquisition, the same odor ' $A$ ' was presented simultaneously in both holes. For recall, the odor first presented during acquisition (odor ' $A$ ') and a novel odor (odor 'B') was presented at randomized positions.

tions of bioanalytes that are susceptible to post-mortem changes can be made. OBs were dissected from the microwave-fixed brain, and the levels of noradrenalin (NA), dopamine (DA), and serotonin (5-HT) were quantified using HPLC with electrochemical detection, using conditions similar to those previously described (Cosi and Marien, 1998). Owing to unsatisfactory tissue fixation, one animal from the OES and three from the OEN-Lab groups were not included in the final analysis. Between-group comparisons were made by ANOVA followed by Bonferroni post hoc tests.

\section{Assessment of Newly Formed Cell Survival and Proliferation}

BrdU administration. On day 20 of the experiment, 24 mice (6 per experimental group) received two injections, $2 \mathrm{~h}$ apart, of 5-bromo-2'deoxyuridine (BrdU; Sigma; $50 \mathrm{mg} / \mathrm{kg}$ in physiological saline) $2 \mathrm{~h}$ before killing, to assess cell proliferation. To assess newborn cell survival, another series of 24 mice ( 6 per experimental group) received also on day
20 three BrdU injections every $2 \mathrm{~h}$ before being returned to their respective cages for the remaining 27 days of the experiment (Figure 1a).

Tissue preparation and sectioning. Mice were deeply anesthetized with pentobarbital $(0.2 \mathrm{ml} / 30 \mathrm{~g}$ body weight $)$ and killed by intracardiac perfusion of a solution containing $4 \%$ paraformaldehyde in $0.1 \mathrm{M}$ phosphate buffer (PB), $\mathrm{pH}$ 7.4. Brains were dissected out and fixed overnight in the same perfusion solution at $4^{\circ} \mathrm{C}$, washed $12 \mathrm{~h}$ in $\mathrm{PB}$, immersed for $48 \mathrm{~h}$ at $4^{\circ} \mathrm{C}$ in $\mathrm{PB}$ containing $20 \%$ sucrose, and frozen in chilled isopentane $\left(-55^{\circ} \mathrm{C}\right)$. The brains were sectioned in the coronal plane with a cryostat (Leica) into $14 \mu \mathrm{m}$ thick serial sections collected at the anatomical levels that comprised the OB and SVZ. The sections were mounted on poly-L-lysine-coated slides (0.05\%; Sigma).

BrdU immunochemistry. Brain sections were incubated in Target Retrieval Solution (Dako, Trappes, France) for $20 \mathrm{~min}$ at $98^{\circ} \mathrm{C}$. After cooling for $20 \mathrm{~min}$, sections were treated with Triton $0.5 \%$ (Sigma; X100) in phosphate- 
buffered saline (PBS) for $30 \mathrm{~min}$, then for $3 \mathrm{~min}$ with pepsin $(0.43 \mathrm{U} / \mathrm{ml}$ in $0.1 \mathrm{~N} \mathrm{HCl}$; Sigma). Endogenous peroxidases were blocked with a solution of $3 \% \mathrm{H}_{2} \mathrm{O}_{2}$ in $0.1 \mathrm{M}$ PBS. Sections were then incubated for $90 \mathrm{~min}$ in $5 \%$ normal horse serum (Vector Laboratories, Burlingame, CA, USA) in 5\% bovine serum albumin (Sigma) and $0.125 \%$ Triton X-100 to block nonspecific binding, and incubated overnight at $4{ }^{\circ} \mathrm{C}$ in a mouse anti-BrdU primary antibody ( $1: 100$; Chemicon, Temecula, CA). Sections were then incubated in a horse biotinylated anti-mouse secondary antibody $(1: 200$; Vector Laboratories) for $2 \mathrm{~h}$. Sections were then processed with avidin-biotin-peroxidase complex (ABC Elite Kit; Vector Laboratories) for $30 \mathrm{~min}$, followed by three rinses of $5 \mathrm{~min}$ in PBS. Finally, sections were reacted in $0.05 \% 3,3^{\prime}$ diaminobenzidine-tetrahydrochloride (Sigma), $0.03 \% \mathrm{NiCl}_{2}$, and $0.03 \% \mathrm{H}_{2} \mathrm{O}_{2}$ in Tris- $\mathrm{HCl}$ buffer $(0.05 \mathrm{M}, \mathrm{pH} 7.6)$, dehydrated in graded ethanols, and coverslipped in DPX.

For double-labeling experiments, sections were treated as described for BrdU immunocytochemistry except that they were incubated simultaneously with rat anti-BrdU (1:100; Oxford Biotechnology, Abcys, Paris) and mouse anti-NeuN antibodies (1:500; Chemicon, Millipore, Billerica, MA, USA) at $4{ }^{\circ} \mathrm{C}$ overnight. Immunoreactivities were revealed with a goat anti-rat antibody conjugated to Alexa 546 and horse anti-mouse conjugated to Alexa 488 (Molecular Probes, Invitrogen, Eugene, OR, USA). For BrdU/Zif268 double labeling, sections were similarly treated except that they were incubated with mouse anti-BrdU (1:100; Chemicon) and rabbit anti-Zif268 antibodies (1:1000; Santa Cruz Biotechnology, Santa Cruz, CA, USA). Secondary antibodies were goat anti-mouse conjugated to Alexa 633 and biotinylated goat anti-rabbit antibodies followed by streptavidin Alexa 488. All the sections were mounted in Vectashield medium with DAPI (Vector Laboratories) and analyzed using a TCS SP2 confocal laser microscope (Leica) at the Centre Commun de Quantimétrie (Université Claude Bernard-Lyon 1). Colocalizations were evaluated in 15-30 cells from each animal $(n=6)$ by performing z-stack acquisitions and three-dimensional reconstructions using QWin (Leica) and Adobe Photoshop version 7.01 (Adobe System, San Jose, CA) software.

BrdU-stained nuclei quantification. Labeled profiles were counted with a Zeiss microscope coupled with a mapping software (Mercator Pro; Explora Nova, La Rochelle, France). All labeled nuclei were counted in the granular layer $(\mathrm{GrL})$ of the $\mathrm{OB}$ ( $\mathrm{six}$ sections per animal, intersection intervals of $336 \mu \mathrm{m})$. The SVZ was delineated at a $\times 40$ objective, based on histological difference from the surrounding tissue around the lateral ventricle and all labeled BrdU-positive cells were counted in three sections per animal starting from the opening of the ventricle (intersection intervals of $336 \mu \mathrm{m}$; Figure $4 \mathrm{c}$ ). The volume of the region of interest was calculated according to a conventional stereological equation (Howard and Reed, 1998) as previously described (Veyrac et al, 2005). The numbers of positive profiles were then related to the volume of the region of interest, to express data as a number of profiles per $\mathrm{mm}^{3}$ (volumetric density).

The data were averaged across animals within each experimental group and statistical differences were assessed using ANOVA followed by Bonferroni post hoc tests.

\section{RESULTS}

\section{Exposure to Novelty is Required for Memory and Neurogenic Effects of Olfactory Enrichment}

Adult mice were submitted to two distinct paradigms of olfactory enrichment differing essentially by the nature of olfactory experience. The first paradigm, known to improve olfactory neurogenesis and memory (Rochefort et al, 2002), consisted of a daily exposure to a new single odorant for 47 days (OEN). In the second paradigm, the same series of odorants was presented as a group every day, thus providing an enriched but stable olfactory environment (OES). Control mice (standard, Stand) were housed in the same conditions of cage size and number of congeners, but were not submitted to any specific olfactory stimulation (Figure 1a; Material and methods).

During the last week of enrichment, short-term memory was assessed in a two-trial odor recognition task conducted on a two-hole board (Veyrac et al, 2007; Figure 1b; Material and methods). During the acquisition trial, exploration time and number of visits were statistically similar in Stand, OEN, and OES groups of animals (Figure 2a) as well the locomotor activity scores (mean distance covered in $\mathrm{cm} \pm \mathrm{SEM}$, Stand $605 \pm 66$; OEN $583 \pm 50$; OES $729 \pm 109$ ). Comparing between groups the time spent exploring the novel or familiar odors during recall revealed that animals from the OES group showed a more variable behavior compared to Stand or OEN (F-test, $p<0.001$ ). However, importantly, overall exploration times of the holes did not differ significantly between groups despite a trend toward longer exploration time of the holes in the OES and OENLab groups (Kruskal-Wallis ANOVA including the four experimental groups, $H=7.1, p=0.07$ for exploration of the familiar odor; $H=6.4, p=0.09$ exploration of the novel odor). The same trends were seen for number of visits $(H=7.5, p=0.55$ and $H=6.1, p=0.1$ for visits to the familiar and novel odor respectively). Thus, any betweengroup difference in odor recollection will not be ascribable to increased exploratory behavior. During recall, the mice of the OEN group were found to spend more time exploring (paired $t$-test, $p=0.03$ ) and to visit more often the hole containing the novel odor ( $p=0.004$; Figure $2 \mathrm{~b}$ ), indicating that they remembered the odorant presented during acquisition. In contrast, mice both in the OES and control groups did not retain this information as they similarly explored the two odorants ( $p>0.05$; Figure $2 \mathrm{~b}$ ).

Consistent with raw data analysis, results expressed as preference for novel odor indicate that the OEN group only explored the novel odor above chance level, both in terms of time and number of visits (one-sample $t$-test, $p=0.004$ and 0.002 , respectively), indicating that only mice from this group could remember the familiarized odor (Figure $3 \mathrm{a}$ and $\mathrm{b}$ ).

Proliferating cells were labeled by injection of animals with BrdU on day 20 (Figure 1a). At the end of the enrichment period, surviving BrdU-labeled cells were counted in the GrL, the main target of olfactory neurogenesis in the OB. The density of BrdU-positive cells was $40 \%$ higher in the OEN group $(\mathrm{F}(3,18)=11.205, p=0.0001$, Bonferroni post hoc, $p<0.005$ for difference between OEN and the other groups) but unchanged in the OES group, compared to control animals (Figure 4a). This effect was not 

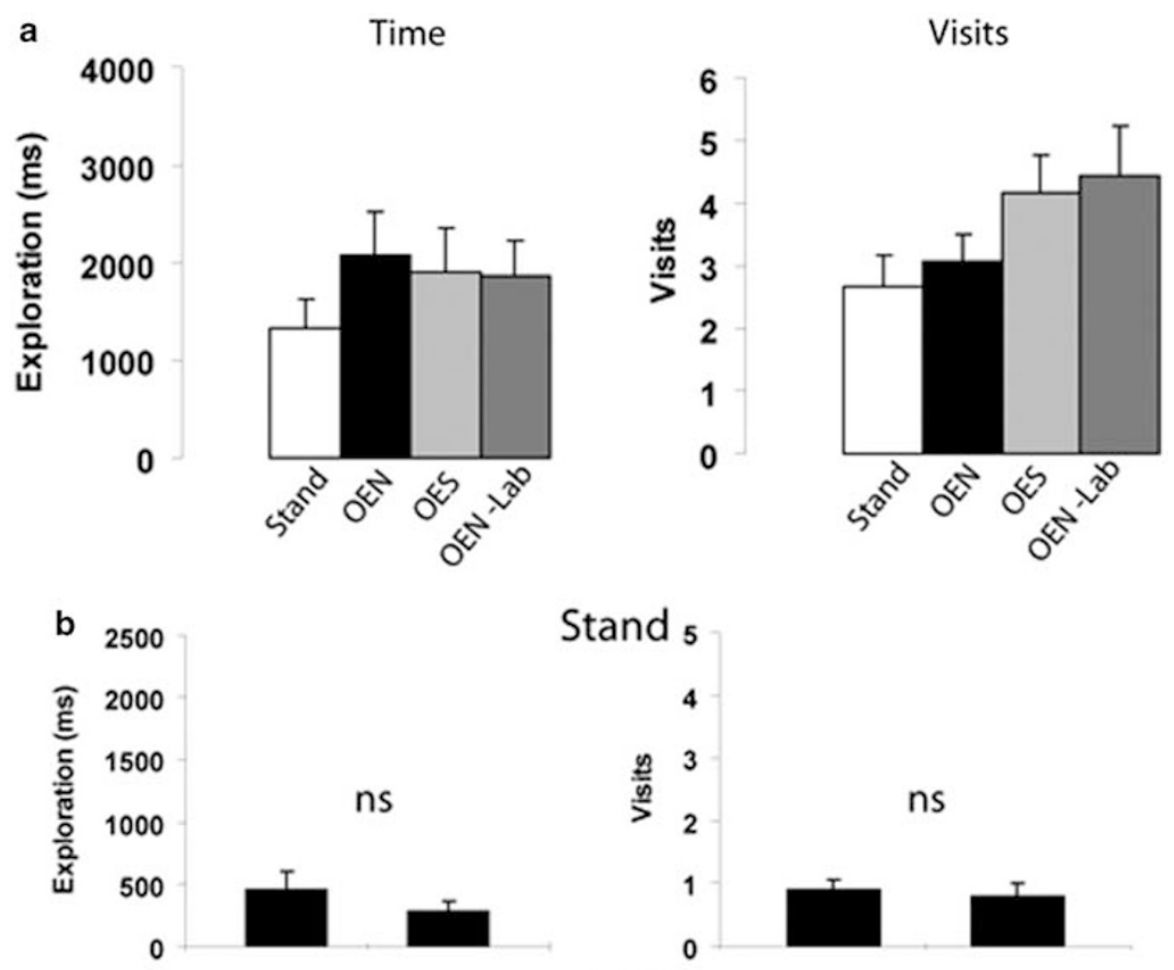

Stand 5

\section{4}

$\begin{array}{ll}\frac{y}{5} & 3 \\ >^{\frac{m}{5}} 2 & \end{array}$

ns
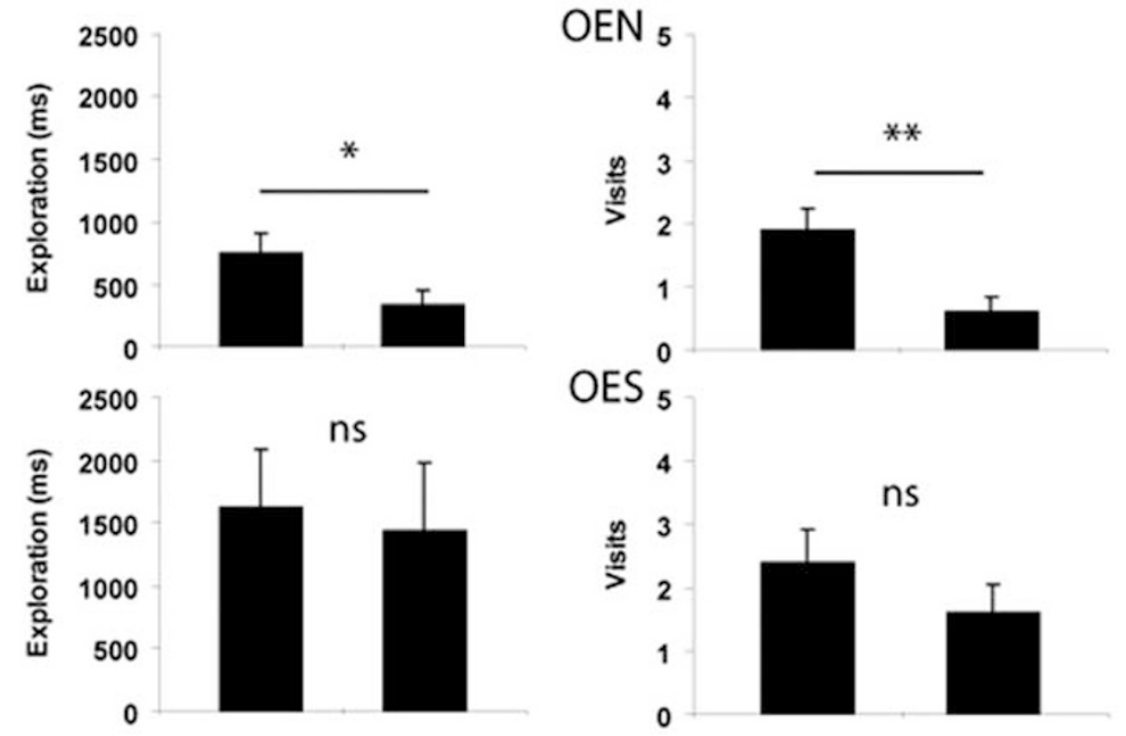

ns
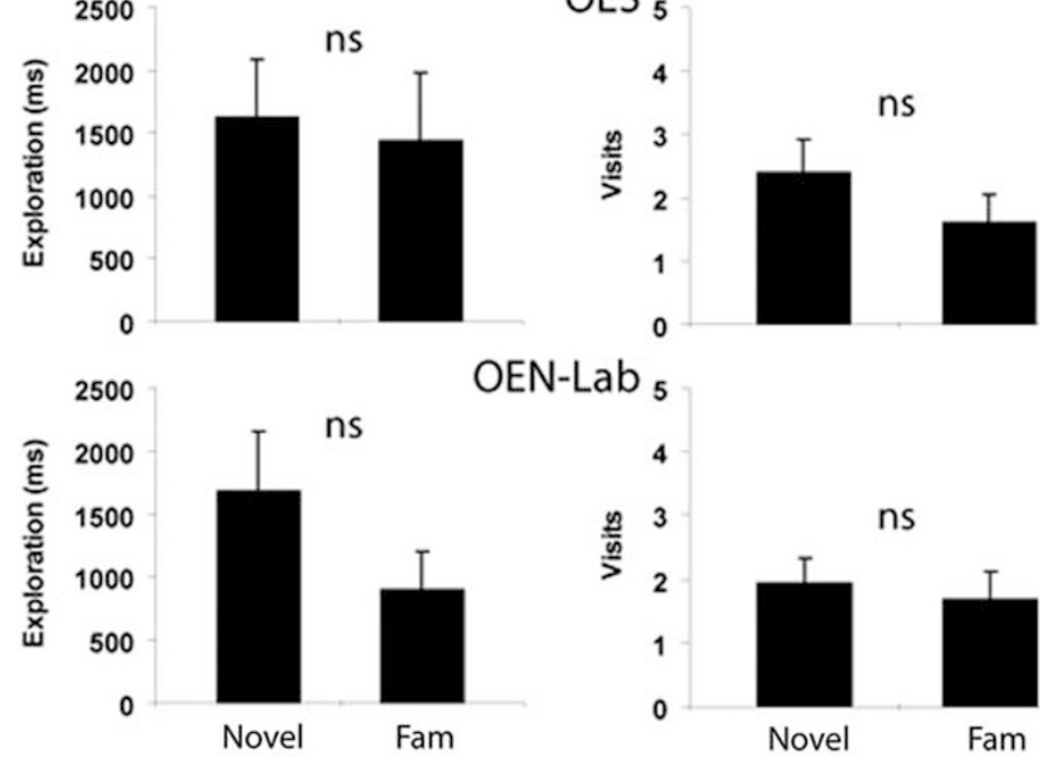

Figure 2 Behavioral effects of olfactory enrichment. (a) Time spent exploring the holes (left panel) and number of visits into the holes (right panel) during the acquisition trial. No difference was found between the experimental groups. (b) Exploration time (left panels) and number of visits into the holes (right panels) containing the novel and familiarized (Fam) odors during recall trial for all experimental groups, as labeled. Only mice of olfactory enrichment/novelty (OEN) group spent more time exploring and visit more often the novel odor compared to the familiarized one (paired $t$-test, $* p<0.05$, *** $<0.005)$. Stand: Standard housing conditions; OES: olfactory enrichment/stable; OEN-Lab: OEN treated with labetalol. Stand, $n=21 ;$ OEN, $n=20 ;$ OES, $n=15 ;$ OEN-Lab, $n=16$ 

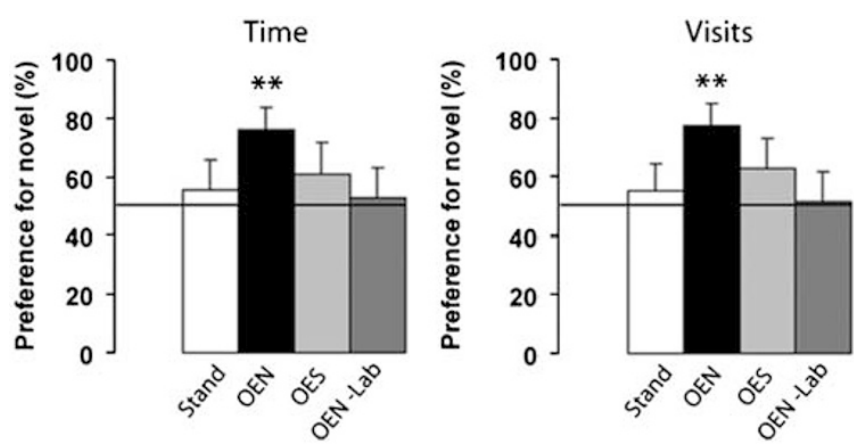

Figure 3 Effects of olfactory enrichment on olfactory memory duration. Exploration time (a) and number of visits (b) of the novel odorant during recall expressed as an index of preference for novel odor. Preference for novel odor was significant for the OEN group only (one sample $t$-test for difference from $50 \%$, ** $<<0.005)$. Stand, standard housing conditions; OEN, olfactory enrichment/novelty; OES, olfactory enrichment/stable; OEN-Lab, OEN treated with labetalol. Stand, $n=21$; OEN, $n=20$; OES, $n=15 ;$ OEN-Lab, $n=16$.

associated with any increase in the rate of proliferation within the SVZ. Indeed, the rate of proliferation measured in the groups of animals killed immediately after BrdU administration was similar in the different olfactory enrichment paradigms (Figure $4 \mathrm{c}$ and $\mathrm{d}$ ). The BrdU-positive cells in the $\mathrm{OB}$ were identified as granule cells by their localization in the GrL (Figure $4 \mathrm{~b}$ ) and by their expression of the neuronal marker NeuN (Rochefort et al, 2002; Figure 4e). The mean percentage of BrdU cells expressing NeuN in animals of the OEN and OES groups $(84.3 \pm 4.7$ and $90.8 \pm 3.4 \%$, respectively) was statistically similar to that of controls $(87.6 \pm 4.6 \%)$. Functional integration of newborn neurons was assessed by the coexpression of BrdU and the immediate-early gene Zif268 (Magavi et al, 2005; Figure 4g). The mean percentage of BrdU/Zif268-positive cells was similar in the experimental groups (Stand, $15.73 \pm 1.74 \%$; OEN, $16.58 \pm 1.81 \%$; and OES, $19.31 \pm 2.55 \%)$, suggesting that the basal level of activation of newborn neurons was similar in the different experimental groups. However, because of the higher number of BrdU-positive cells retained in the $\mathrm{OB}$, the density of BrdU-Zif268 was significantly higher in the OEN group compared to control or OES group $(\mathrm{F}(3,11)=6.87, p=0.007$, Bonferroni post hoc, $p<0.05$ for difference between OEN and the other groups) (Figure $4 \mathrm{f}$ ).

\section{The Level of NA in the OB is Enhanced by Repeated Exposure to Novelty}

To test the hypothesis that the OEN paradigm activates the noradrenergic input to the $\mathrm{OB}, \mathrm{NA}$ levels in the $\mathrm{OB}$ were measured by HPLC (see Material and methods). Compared to standard animals, NA levels were increased in the $\mathrm{OB}$ of the OEN but not of the OES group $(F(3,16)=11.07$, $p=0.0001$ Bonferroni post hoc, $p<0.05$ for difference between OEN and all other groups) (Figure $5 \mathrm{a}$ ). In contrast, the levels of DA (a neurotransmitter intrinsic to the OB and whose expression is regulated by olfactory input) and 5-HT (another centrifugal monoaminergic system innervating the $\mathrm{OB}$ ) were found unchanged in the same tissue (Figure $5 \mathrm{~b}$ and $c$ ).

\section{The NA Receptor Antagonist Labetalol Blocks the Effects of Enrichment}

To further document a role of NA in mediating the effects of environmental novelty, the OEN condition was applied to an independent group of mice treated orally with the mixed $\beta$ - and $\alpha 1$-adrenoceptor antagonist, labetalol (Goodman Gilman et al, 1991) (OEN-Lab group; Figure 1a), to block the postsynaptic actions of NA mediated by these receptors. In contrast to animals in the OEN group, the OEN-Lab mice showed no increase either in the density of BrdU-positive cells (Figure 4a) or in the density of BrdU-Zif268 co-labeled cells in GrL (Figure 4f), and performed at chance level in the odor recognition task (Figures $2 \mathrm{~b}$ and 3 ). In addition, the level of NA in the $\mathrm{OB}$ was not increased in the OEN-Lab group (Figure 5a), most likely because of a blockade by labetalol of excitatory $\alpha 1$-autoreceptors on LC-noradrenergic neurons (Pudovkina and Westerink, 2005). Finally, the treatment with labetalol did not affect any of the following parameters: exploration of the holes (Figure 2a and b), motor activity ( $500 \pm 53 v s 583 \pm 48$ for OEN, mean distance covered \pm SEM in $\mathrm{cm}$ ), rate of neuronal differentiation in the OB $(90.3 \pm 1.4 \%$ of BrdU-NeuN double-labeled cells in OEN-Lab vs $84.3 \pm 4.8 \%$ in OEN), rate of proliferation in the SVZ (Figure 4d), mean percentage of BrdU/Zif268-positive cells (OEN-Lab, $17.95 \pm 4.13$ vs OEN, $16.58 \pm 1.81$ ), and levels of DA and 5-HT in the OB (Figure 5b and c).

\section{DISCUSSION}

In the present study, we used the same set of odorants in two different ways to isolate the contribution of novelty to the effect of olfactory enrichment on OB plasticity. Our data indicate that in contrast to repeated exposure to novel odors alone, which was sufficient for increasing bulbar neurogenesis and short-term olfactory memory duration, exposure to an olfactory-enriched environment consisting of several odors that were not renewed in the environment did not stimulate olfactory neurogenesis or increase memory duration. Importantly, the basal level of activation of the $\mathrm{OB}$ as evidenced by the level of BrdU/Zif268 expression was similar in OEN and OES animals even though OEN animals were exposed to one odor at time $v s$ several odors for the OES animals. This might be explained by the fact that we used complex odors, each of which is likely to produce a broad activation of the OB network. Differences between OEN and OES groups can thus most likely not be ascribed to overall differences in activation of the $\mathrm{OB}$. Exposure to novelty thus appears as a key parameter underlying the effects of olfactory enrichment on neurogenesis and olfactory short-term memory. This may conciliate previous studies wherein a procedure of olfactory enrichment that consisted of repeated exposure to similar odorants did not increase neurogenesis (Magavi et al, 2005) whereas repeated exposure to new odors did (Rochefort et al, 2002). Repeated exposure to odorants has been shown to affect olfactory processing significantly as evidenced by improved discrimination of perceptually similar odorants (Mandairon et al, $2006 \mathrm{~b}, \mathrm{c})$. Interestingly, in this paradigm, odor exposure consisted of $20 \mathrm{~min}$ exposure per day, leaving the possibility that the odors used for enrichment appear novel every day. This form of enrichment could thus also involve NA 

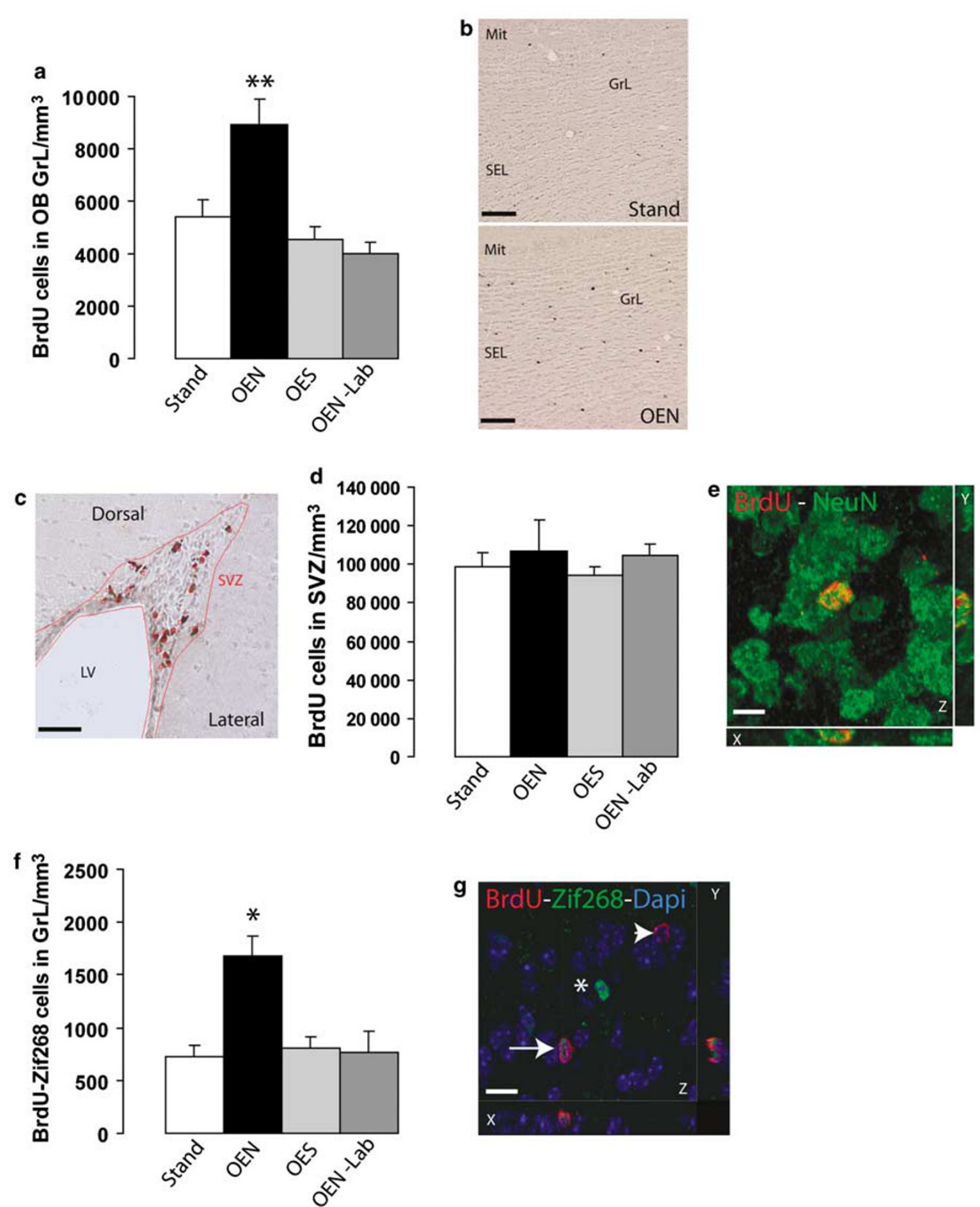

Figure 4 Cellular effects of olfactory enrichment. (a) Density of 5-bromo-2'deoxyuridine (BrdU)-positive cells in the granular cell layer (GrL) of the olfactory bulb $(\mathrm{OB})$ in the four experimental groups, measured 47 days after BrdU administration. The OEN group showed significantly more labeled cells than the other groups (ANOVA followed by Bonferroni post hoc *** $<0.005$ for difference between OEN and the other groups). Stand, $n=6$; OEN, $n=4$; OES, $n=6$; OEN-Lab, $n=6$. (b) BrdU-positive cells located in the GrL of the OB in control and OEN animals. Mit, mitral cell layer; SEL, subependymal layer; scale bar $=60 \mu \mathrm{m}$. (c) BrdU-positive cells in the subventricular zone (SVZ) analyzed $2 \mathrm{~h}$ post-BrdU administration on day 20. LV, lateral ventricle; scale bar $=40 \mu \mathrm{m}$. (d) Density of BrdU-positive cells in the SVZ in the four experimental groups. Densities are similar in all four experimental groups. Stand, $n=5$; OEN, $n=5$; OES, $n=6$; OEN-Lab, $n=6$. (e) Representative micrograph of a BrdU/NeuN double-labeled cell under confocal microscopy. Scale bar $=5 \mu$ m (f) Density of BrdU/Zif268-positive cells in the GrL is increased in OEN compared to the other groups (ANOVA followed by Bonferroni post hoc *p <0.05 for difference between OEN and the other groups). (g) Representative micrograph of BrdU/Zif268 double-labeling showing a BrdU/Zif268-positive cell (arrow), a BrdU-positive and Zif268-negative cell (arrowhead) and a Zif268-positive and BrdU-negative cell (asterisk). Scale bar = $10 \mu \mathrm{m}$. Stand, standard housing conditions; OEN, olfactory enrichment/novelty; OES, olfactory enrichment/stable; OEN-Lab, OEN treated with labetalol. 

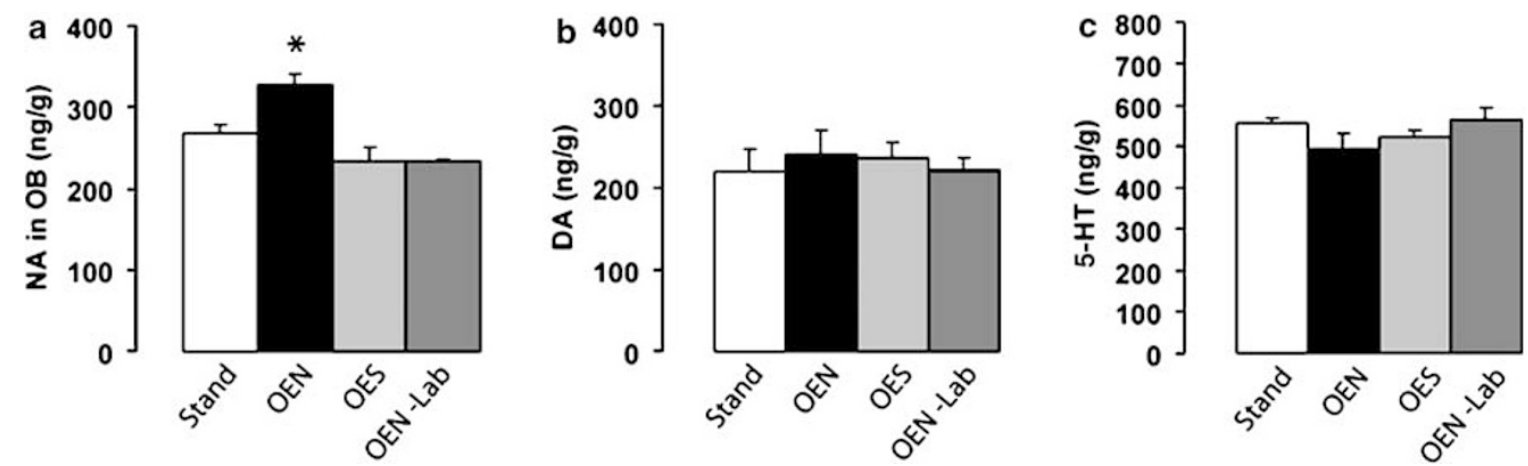

Figure 5 Effects of olfactory enrichment on neurotransmitters levels. Tissue concentration of (a) noradrenalin (NA), (b) dopamine (DA), and (c) serotonin (5-HT). NA is significantly increased in the OEN group compared to the standard condition group (ANOVA followed by Bonferroni post hoc * $p<0.05$ for difference between OEN and all other groups; $p>0.05$ for all other comparisons). 5-HT and DA levels are unchanged. Group labels as in Figure 4. Stand, $n=6$; OEN, $n=6$; OES, $n=5$; OEN-Lab, $n=3$.

mechanisms and modulate neurogenesis. Further studies will be required to test this hypothesis.

The increase in the number of newborn neurons observed in the OEN group can be ascribed to better survival. Indeed, first, proliferation rate of progenitors in the SVZ is not affected by enrichment. Second, enrichment is applied during the critical period when many newborn neurons die unless they are rescued by sensory inputs (Winner et al, 2002; Yamaguchi and Mori, 2005; Mandairon et al, 2006a).

In a second part of the study, we provide evidence for the involvement of NA-dependent mechanisms underlying enrichment-induced plasticity. We first found an increase in the $\mathrm{OB}$ tissue concentration of NA in the OEN group. This increase in NA level was not associated with a general enhancement in neurotransmitter levels in the $\mathrm{OB}$ in response to enrichment as those of 5-HT and DA were unaffected in the same tissue samples. In addition, the treatment by the mixed $\beta$ - and $\alpha 1$-adrenoceptor antagonist labetalol blocked the effect of olfactory enrichment. Importantly, the labetalol treatment did not affect the level of exploration of the odors, nor the general locomotor activity of the animals. Thus, the ability of labetalol to block the effects of olfactory enrichment cannot be attributed to a lower interest and/or rate of exploration of novel odors. These findings support a role for central NA mechanisms in mediating the effects of novelty-dependent olfactory enrichment.

Within the $\mathrm{OB}$, noradrenergic fibers terminate densely, although not exclusively, on granule cells (Shipley et al, 1985; Mandairon and Didier, 2008) providing an anatomical substrate by which NA influence a variety of olfactory learning (Brennan and Keverne, 1997; Brennan et al, 1998; Sullivan et al, 2000; Veyrac et al, 2007). Furthermore, increased LC activity is observed during processing of novel sensory stimuli (Foote et al, 1980; Aston-Jones and Bloom, 1981; Bouret and Sara, 2005), suggesting that NA participates in processing novel stimuli and familiarization. Increased NA level in OEN group could thus reflect repeated activation of the LC with daily exposure to novel odors; this would not occur in the OES group because it does not experience novelty. In addition, NA has been shown to be a positive regulator of neurogenesis both on the dentate gyrus, the other main neurogenic region of the brain (Kulkarni et al, 2002; Rizk et al, 2006), and the OB (Bauer et al, 2003; Veyrac et al, 2005). Although its molecular mechanism of action remains unknown, NA has been shown to be neuroprotective in lesion models such as ischemia or devascularization (Martel et al, 1998; Debeir et al, 2002). On the basis of the increased survival of newborn neurons upon enrichment and its blockade by an NA antagonist, our data suggest that NA exerts its neuroprotective effect also on adult-born neurons in the OB. The increased survival induced by olfactory enrichment has been shown to be transient, the newborn cells rescued by enrichment disappearing from the OB 7 weeks after enrichment (Rochefort and Lledo, 2005). This suggests that NA could be necessary not only to rescue newborn cells arriving in the $\mathrm{OB}$ but would also be useful for their longterm survival. If true, this hypothesis would predict that a prolonged enrichment maintains the rescued cells in place, but this remains to be confirmed.

By showing a parallel enhancement of neurogenesis and memory performance in the OEN paradigm, our data confirm the correlative correlation between changes in neurogenesis and memory performance (Rochefort et al, 2002; Alonso et al, 2006; Mandairon et al, 2006d). Furthermore, because of an increase in the number of BrdU cells in the OEN group, the density of newborn (BrdU positive) cells co-labeled by Zif268 was higher than that of all of the other experimental groups. Thus, in the particular group where the effects of enrichment are observed, more newborn neurons participate in the bulbar network, providing a potential mechanism linking an increased survival of newborn cells and enhanced olfactory memory performances. Indeed, more newborn cells incorporated into the bulbar network may have contributed to improved odor processing in the $\mathrm{OB}$ (Cecchi et al, 2001) and to the formation of olfactory memory itself (Alonso et al, 2006; Mandairon et al, 2006d).

Finally, combining our current results with previous data showing improvement in neurogenesis following associative learning, one may suggest that neurogenesis increases in the $\mathrm{OB}$ in response to stimuli that are salient, whether because they are novel (current data) or because they are predictive of a reward (Alonso et al, 2006; Mandairon et al, 2006d). In the latter case, neuromodulatory systems such as the cholinergic system may be preferentially involved but this hypothesis remains to be tested. 
In recent years, experimental studies have repeatedly shown that environmental enrichment can improve behavioral, cellular, and molecular outcomes in models of human central nervous system disorders (Nithianantharajah and Hannan, 2006) that include Alzheimer's disease and Parkinson's disease, fragile X and Down's syndromes, and various forms of brain injury. Our demonstration that novelty is a critical factor determining the consequences of olfactory enrichment, and that the noradrenergic system is a key stimulus-dependent mediator of memory and neurogenic changes induced by repeated novelty recognition, may have potentially important implications for novel therapeutic strategies in the treatment of these disorders.

\section{ACKNOWLEDGEMENTS}

This work was supported by CNRS, Université Claude Bernard-Lyon 1 and Région Rhônes-Alpes (fellowship to $\mathrm{AV})$.

\section{DISCLOSURE/CONFLICT OF INTERESTS}

The authors declare no conflict of interest.

\section{REFERENCES}

Alonso M, Viollet C, Gabellec MM, Meas-Yedid V, Olivo-Marin JC, Lledo PM (2006). Olfactory discrimination learning increases the survival of adult-born neurons in the olfactory bulb. J Neurosci 26: 10508-10513.

Aston-Jones G, Bloom FE (1981). Norepinephrine-containing locus coeruleus neurons in behaving rats exhibit pronounced responses to non-noxious environmental stimuli. J Neurosci 1: 887-900.

Bauer S, Moyse E, Jourdan F, Colpaert F, Martel JC, Marien M (2003). Effects of the alpha 2-adrenoreceptor antagonist dexefaroxan on neurogenesis in the olfactory bulb of the adult rat in vivo: selective protection against neuronal death. Neuroscience 117: 281-291.

Bennett EL, Rosenzweig MR, Diamond MC (1969). Rat brain: effects of environmental enrichment on wet and dry weights. Science 163: 825-826.

Bouret S, Sara SJ (2005). Network reset: a simplified overarching theory of locus coeruleus noradrenaline function. Trends Neurosci 28: 574-582.

Brennan PA, Keverne EB (1997). Neural mechanisms of mammalian olfactory learning. Prog Neurobiol 51: 457-481.

Brennan PA, Schellinck HM, de la Riva C, Kendrick KM, Keverne EB (1998). Changes in neurotransmitter release in the main olfactory bulb following an olfactory conditioning procedure in mice. Neuroscience 87: 583-590.

Cecchi GA, Petreanu LT, Alvarez-Buylla A, Magnasco MO (2001). Unsupervised learning and adaptation in a model of adult neurogenesis. J Comput Neurosci 11: 175-182.

Cosi C, Marien M (1998). Decreases in mouse brain NAD+ and ATP induced by 1-methyl-4-phenyl-1, 2,3,6-tetrahydropyridine (MPTP): prevention by the poly(ADP-ribose) polymerase inhibitor, benzamide. Brain Res 809: 58-67.

Debeir T, Marien M, Chopin P, Martel JC, Colpaert F, RaismanVozari R (2002). Protective effects of the alpha 2-adrenoceptor antagonist, dexefaroxan, against degeneration of the basalocortical cholinergic system induced by cortical devascularization in the adult rat. Neuroscience 115: 41-53.

Foote SL, Aston-Jones G, Bloom FE (1980). Impulse activity of locus coeruleus neurons in awake rats and monkeys is a function of sensory stimulation and arousal. Proc Natl Acad Sci USA 77: 3033-3037.

Goodman Gilman A, Rall T, Nies A, Taylor P (1991). The Pharmacological Basis of Therapeutics. Pergamon Press: New York, NY.

Howard C, Reed M (1998). Unbiased Stereology: Three-Dimensional Measurement in Microscopy. Bios Scientific: Oxford, UK.

Kulkarni VA, Jha S, Vaidya VA (2002). Depletion of norepinephrine decreases the proliferation, but does not influence the survival and differentiation, of granule cell progenitors in the adult rat hippocampus. Eur J Neurosci 16: 2008-2012.

Lledo PM, Alonso M, Grubb MS (2006). Adult neurogenesis and functional plasticity in neuronal circuits. Nat Rev Neurosci 7: 179-193.

Magavi SS, Mitchell BD, Szentirmai O, Carter BS, Macklis JD (2005). Adult-born and preexisting olfactory granule neurons undergo distinct experience-dependent modifications of their olfactory responses in vivo. J Neurosci 25: 10729-10739.

Mandairon N, Didier A (2008). Neuromodulation in the main olfactory bulb. Encyclopedia of Neuroscience. Springer: New York.

Mandairon N, Sacquet J, Jourdan F, Didier A (2006a). Long-term fate and distribution of newborn cells in the adult mouse olfactory bulb: influences of olfactory deprivation. Neuroscience 141: 443-451.

Mandairon N, Stack C, Kiselycznyk C, Linster C (2006b). Enrichment to odors improves olfactory discrimination in adult rats. Behav Neurosci 120: 173-179.

Mandairon N, Stack C, Kiselycznyk C, Linster C (2006c). Broad activation of the olfactory bulb produces longlasting changes in odor perception. Proc Natl Acad Sci USA 103: 13543-13548.

Mandairon N, Sacquet J, Garcia S, Ravel N, Jourdan F, Didier A (2006d). Neurogenic correlates of an olfactory discrimination task in the adult olfactory bulb. Eur J Neurosci 24: 3578-3588.

Martel J, Chopin P, Colpaert F, Marien M (1998). Neuroprotective effects of the alpha2-adrenoceptor antagonists, $(+)$-efaroxan and $(+/-)$-idazoxan, against quinolinic acid-induced lesions of the rat striatum. Exp Neurol 154: 595-601.

Nithianantharajah J, Hannan AJ (2006). Enriched environments, experience-dependent plasticity and disorders of the nervous system. Nat Rev Neurosci 7: 697-709.

Pudovkina OL, Westerink BH (2005). Functional role of alpha1adrenoceptors in the locus coeruleus: a microdialysis study. Brain Res 1061: 50-56.

Rizk P, Salazar J, Raisman-Vozari R, Marien M, Ruberg M, Colpaert $\mathrm{F}$ et al (2006). The alpha2-adrenoceptor antagonist dexefaroxan enhances hippocampal neurogenesis by increasing the survival and differentiation of new granule cells. Neuropsychopharmacology 31: 1146-1157.

Rochefort C, Lledo PM (2005). Short-term survival of newborn neurons in the adult olfactory bulb after exposure to a complex odor environment. Eur J Neurosci 22: 2863-2870.

Rochefort C, Gheusi G, Vincent JD, Lledo PM (2002). Enriched odor exposure increases the number of newborn neurons in the adult olfactory bulb and improves odor memory. J Neurosci 22: 2679-2689.

Rosenzweig MR, Bennett EL (1996). Psychobiology of plasticity: effects of training and experience on brain and behavior. Behav Brain Res 78: 57-65.

Schrijver NC, Bahr NI, Weiss IC, Wurbel H (2002). Dissociable effects of isolation rearing and environmental enrichment on exploration, spatial learning and HPA activity in adult rats. Pharmacol Biochem Behav 73: 209-224.

Schrijver NC, Pallier PN, Brown VJ, Wurbel H (2004). Double dissociation of social and environmental stimulation on spatial learning and reversal learning in rats. Behav Brain Res 152: $307-314$. 
Shipley MT, Halloran FJ, de la Torre J (1985). Surprisingly rich projection from locus coeruleus to the olfactory bulb in the rat. Brain Res 329: 294-299.

Sullivan RM, Stackenwalt G, Nasr F, Lemon C, Wilson DA (2000). Association of an odor with activation of olfactory bulb noradrenergic beta-receptors or locus coeruleus stimulation is sufficient to produce learned approach responses to that odor in neonatal rats. Behav Neurosci 114: 957-962.

van Praag H, Kempermann G, Gage FH (2000). Neural consequences of environmental enrichment. Nat Rev Neurosci 1: 191-198.

van Praag H, Christie BR, Sejnowski TJ, Gage FH (1999). Running enhances neurogenesis, learning, and long-term potentiation in mice. Proc Natl Acad Sci USA 96: 13427-13431.

Vankov A, Herve-Minvielle A, Sara SJ (1995). Response to novelty and its rapid habituation in locus coeruleus neurons of the freely exploring rat. Eur J Neurosci 7: 1180-1187.
Veyrac A, Didier A, Colpaert F, Jourdan F, Marien M (2005). Activation of noradrenergic transmission by alpha2-adrenoceptor antagonists counteracts deafferentation-induced neuronal death and cell proliferation in the adult mouse olfactory bulb. Exp Neurol 194: 444-456.

Veyrac A, Nguyen V, Marien M, Didier A, Jourdan F (2007). Noradrenergic control of odor recognition in a nonassociative olfactory learning task in the mouse. Learn Mem 14: 847-854.

Winner B, Cooper-Kuhn CM, Aigner R, Winkler J, Kuhn HG (2002). Long-term survival and cell death of newly generated neurons in the adult rat olfactory bulb. Eur J Neurosci 16: 1681-1689.

Yamaguchi M, Mori K (2005). Critical period for sensory experience-dependent survival of newly generated granule cells in the adult mouse olfactory bulb. Proc Natl Acad Sci USA 102: 9697-9702. 